\title{
Una alfabetización científica tecnológica y cultural
}

\author{
A technological and cultural scientific alphabetization
}

Adriana Patricia Gallego Torres

Jair Zapata $\mathbf{P}^{2}$

Monica Rueda Pinto ${ }^{3}$

\section{Resumen}

En este artículo se discute la necesidad de una alfabetizacion cientifica tecnologica y cultural para el nuevo milenio, orientada a mitigar los retos ambientales, sociales y culturales de nuestro tiempo. Esta alfabetizacion, ha de estar vertebrada por las relaciones ctsc (ciencia, tecnologia, sociedad y cultura) y fundamentada desde las reconstrucciones de la historia social de las ciencias y de las tecnologías, asi como tambien, en la sociologia de la ciencias y las tecnologías.

\begin{abstract}
In this article there is discussed the need of a scientific technological and cultural literacy for the new millenium, orientated to mitigating the environmental, social and cultural challenges of our time. This literacy, it has to be vertebrada for the relations ctsc (science, technology, society and culture) and based from the reconstructions of the social history of the sciences and of the technologies, this way like also, in the sociology of the ciencias and the technologies.
\end{abstract}

\section{Palabras clave:}

Historia social de las ciencias, relaciones CTSC, didáctica de las ciencias.

\section{Key words:}

Social History of the Sciences, Relations CTSC, Sciences Education.

\section{Introducción}

Para comenzar, debemos tomar una postura sobre lo que consideramos o a lo que nos referimos cuando hablamos de alfabetización científica y tecnológica.

1 Profesora del Doctorado en Educación de la Universidad Distrital Francisco José de Caldas. Bogotá, D. C. E-mail: adpgallegot@ udistrital.edu.co

2 Estudiante del Doctorado en Educación de la Universidad Distrital Francisco José de Caldas. Bogotá, D. C. e-mail: jzapata25@gmail. com. Grupo de investigación en Ensenanza de las Ciencias y las Matemáticas- ENCIMA.

3 Profesora Universidad Santo Tomas. Grupo de investigación en Ensenanza de las Ciencias y las Matemáticas- ENCIMA 
El concepto de alfabetización científica puede remontarse a la década de los años 60. introducido por Paul DeHart Hurd, pero realmente adquiere relevancia a finales del siglo $\mathrm{xx}$ y es allí cuando la comunidad académica comienza a plantearse la necesidad de alfabetizar científica y tecnológicamente a los ciudadanos (Bybee y DeBoer, 1994; Bybee, 1997; Marco, 2000; Fensham, 2002, Gallego Torres, 2002; Vilches, Solbes y Gil 2004).

La alfabetización científica y tecnológica es mucha más que una analogía tomada de la alfabetización iniciada a finales del siglo xix, la cual tenía como objetivo primordial la práctica elemental de la lectura y la escritura adquirida por las grandes mayorías, que no era otra cosa que la expansión social de la lengua escrita (Braslavsky, 2004).

De la misma manera, el movimiento de alfabetización científico tecnológica, inicialmente tenia como objeto la expansión social de la ciencia y la tecnología y promovía la inclusión en el currículo de ciencias de actividades que le permitieran a los estudiantes la comprensión del carácter social de la ciencia y la tecnología, con vistas a favorecer la participación ciudadana en la toma fundamentada de decisiones (Bybee y DeBoer, 1994; Bybee, 1997; Marco, 2000; Vilches, Solbes y Gil, 2004).

Dados los graves problemas a los que se enfrenta la humanidad, nosotros planteamos una alfabetización científica y tecnológica que implique un cambio cultural en este sentido, "una alfabetización científico tecnológica y cultural". No se trata, entonces, solamente de promover la educación científica y tecnológica, de dar a conocer la mayor cantidad posible de conceptos, leyes o teorías que en muchos casos se presentan totalmente descontextualizados y alejados de la vida real, sino de promover una formación científica tecnológica y cultural, ya que estamos convencidos que para que la humanidad pueda afrontar los problemas derivados del abuso del hombre en su intento por dominar la naturaleza, se necesita una verdadera formación ciudadana, basada en un cambio radical en la manera en la que nos relacionamos con ella. Esto nos llevaría a plantearnos una visión distinta a la que poco a poco los productos de la ciencia y la tecnología nos llevaron, es decir, promover una alfabetización que por un lado, lleve a los ciudadanos a comprender e interactuar de modo racional con la realidad cotidiana y, por otro, a ser capaces de tomar decisiones conscientes y responsables ante los graves problemas que se nos avecinan.

Este nuevo enfoque que incluye no solamente a la educación en ciencias, planteada en términos de las relaciones СTs, en virtud de la conciencia que se tiene que es necesario un movimiento internacional con miras a preservar el planeta tierra para las futuras generaciones, frente a las posibles catástrofes naturales que podrían suceder. Es dentro de tal movimiento que se ha propuesto una alfabetización científica, tecnológica y cultural, como un derecho emergente pero orientado no solo a la escuela, sino también a los ambientes formales e informales, ya que para poder apuntar al cambio cultural de los ciudadanos debemos actuar desde distintos frentes y a distintos públicos.

\section{La historia social de las ciencias como fundamento para una nueva albatizacion cientifico tecnologica y cultural}

Es necesario recordar que las relaciones CTS, emergieron de los estudios sociales de las ciencia, particularmente a partir de los trabajos R. K. Merton, Science, technology, and society in seventeen century England, de (1938, 1949), y Theory and social structure, de 1949 (Barona, 1994). La historia social de la ciencia como campo de estudios, recupera el convencimiento que la ciencia hace parte de la sociedad y han estado ligadas a la cultura, en cada una de las épocas de su desarrollo. Se opone a versiones históricas y filosóficas que legitiman una imagen de esta ciencia que la califica como la más genuina expresión de la racionalidad, esto es, sus elaboraciones son asumidas como el conocimiento por excelencia (Eslava, 2004). Destacan estos estudios que la ciencia de la naturaleza es un fenómeno social, es decir, el conocimiento científico es una construcción social, por lo que son comprensibles las investigaciones de las relaciones entre ciencia, tecnología y sociedad (Restivo, 1992). 
Se afirma, por tanto, que el origen y desarrollo de la ciencia moderna se halla estrechamente ligado al origen y desarrollo de la sociedad contemporanea, por lo que tal ciencia se encuentra fundamentalmente ligada a la tecnología moderna, a los valores, intereses y estructuras básicas del capitalismo. Es así, por cuanto esa ciencia surgió en el marco del capitalismo y se institucionalizó como el modelo cognoscitivo de la industrialización. No es extraño suponer que llegó a ese mundo como un bien de consumo y que se desarrolló en íntima relación con los constructores de tecnología. Una de las críticas planteadas es la que se ha dirigido al mito de una ciencia pura, derivada de actos mentales aislados, que se producen en la contemplación pura; contemplación que se sustenta en la búsqueda del conocimiento por sí mismo. Esta creencia sostiene que no está contaminada por actos sociales ni por la cultura en la que es producida dicha creación. Se agrega en contra, que si bien algunos científicos trabajan impulsados por nobles motivaciones, lo que producen sirve a intereses sociales (Restivo, 1992).

Los interrogantes que se encuentran en la base de estos estudios, son los que persiguen dar cuenta de cómo surgió esta ciencia y de qué manera se instituyó como tradición, cómo se organizó la investigación, cuáles son han sido los factores qué han determinado los cambios en la organización científica y cómo se relacionan estos cambios con la investigación. Todos estos interrogantes inscritos en el problema de qué es lo que ha hecho que el conocimiento científico elaborado se haya constituido como único entre las instituciones productoras de cultura y de qué manera elaborar una explicación admisible en torno a los orígenes de la física en el siglo xvii y su ascenso en cuatro siglos a una posición de monopolio cognitivo sobre ciertas esferas de decisión (Vessuri, 1992). Se puntualiza también, que el pensamiento y la práctica científicos han desempeñado un papel decisivo en la historia y la cultura de occidente, por lo que en los estudios históricos ha dominado una versión eurocentrista; versión esta que ha marginado los análisis de las posibles contribuciones de los científicos de los denominados países del tercer mundo (Eslava, 2004).

\section{El componente epistemologico de la alfabetizacion cientifica tecnologica y cultural}

Las relaciones entre epistemología, ciencia, tecnologia y sociedad tienen una compleja historia de la que no es posible dar aquí una visión detallada dada la intencion de este trabajo y dados los tratados que sobre epistemologia de la ciencia y la tecnología existen, donde se profundiza entorno a la epistemología misma. Solamente, nos referiremos a algunos de los cambios paradigmáticos del siglo pasado que han dejado su huella en la historia social de la educación en ciencias y tecnolgías, ya que estamos convencidos que para poder realmente hablar de cambios significativos en la eduación en ciencias y tecnologías se hace necesario acudir a esta categoría, un poco escondida detrás de la historia social de la ciencias, pero que aqui aprovechamos la ocasión para reivindicar la necesidad de promover reflexiones conjuntas acerca de incluir en nuestros discursos la historia social de la educación en ciencias como parte de la historia social de la ciencia (Gallego, R; Gallego, A; Perez, R y Figueroa R, 2009). Una vez aclarado esto, comezaremos por referirnos a algunos paradigmas epistemológicos predominantes.

Una historia de las propuestas epistemológicas muestra que, primero, se contó con la empirista de F. Bacon, dada a conocer en 1620 y fundada en la lógica inductiva (Bacon, 1980); luego fue paulatinamente sustituida por la positivista, desarrollada por Auguste Comte entre 1830 y 1844 (Comte, 1984), igualmente basada en la lógica inductiva y que dominó entre los especialistas hasta las cuatro décadas iniciales del siglo xx. Bacon propone el método de las tres tablas; los positivistas formulan el denominado método científico. La aproximación positivista fue, igualmente, la que puso a circular la idea de que las tecnologías eran una aplicación de las leyes descubiertas por los científicos a los procesos de producción (Comte, 1984).

Todo cambió a partir de 1905, con los artículos de Einstein, de Heisemberg en 1925 y de Schrödinger en 1926, para solo mencionar a los protagonistas más conocidos. El primero con sus mecánicas relativistas acotó la dinámica newtoniana y los se- 
gundos pusieron el tela de juicio al determinismo filosófico; lo que generó la controvertida posición de Einstein, específicamente con la interpretación de Bohr de la ecuación de ondas. La especial revolución que estos artículos produjeron en la física y en la concepción de mundo que venía dominando entre los practicantes de esta ciencia, hicieron que fuera necesario volver a preguntarse por el carácter del conocimiento científico.

La primera respuesta fue dada en 1935, cuando Karl Popper publica su Logik der Forshung (Popper, 1962), en la que establece que desde la lógica inductiva no es posible explicar dicha revolución, retoma entonces la deductiva y la categoría epistemológica de teoría tal como había sido fundada por Newton. Puntualizará que la labor de los hombres de ciencias no es la de hacer observaciones, sino la de formular proposiciones y sistemas de proposiciones (teorías) que han de contrastar sistemáticamente. Si con el positivismo la historia del desarrollo científico es una sucesión lineal de descubrimiento, a partir de Popper se entenderá como un proceso de sustitución de teorías, fuera de que la aceptación de una teoría obedece a una aceptación intersubjetiva de pares. Dirá que la preocupación por la verdad es asunto de los metafísicos y no de los científicos.

Desde la misma posición deductivista, Thomas S. Kuhn en 1962 elabora otra explicación, acudiendo a las categorías de teoría, paradigma, ciencia normal, crisis paradigmática, ciencia revolucionaria y comunidad científica (Kuhn, 1972). Este es conocido como epistemólogo-historicista, ya que es quien busca zanjar la polémica entre los internalistas, los que se mantenían en la postura de que los análisis epistemológicos debían basarse en la lógica, y los externalistas que defendían la necesidad de una historia social de la ciencia. Esto lo logra al proponer que todo estudio epistemológico ha de basarse en revisiones históricas. El desarrollo histórico del conocimiento científico es explicado en Kuhn como un proceso de cambios paradigmáticos; cambios estos en los que la actividad científica vuelve a comenzar y, por tanto, no habrá ese punto final en el que se alcance una explicación verdadera y definitiva de la naturaleza.
En esta misma perspectiva, Imre Lakatos (1983), crítico de Popper y de Kuhn, da cuenta de dicha revolución acudiendo a la categoría de programa de investigación científica. Define tal categoría con las también categorías epistemológicas de núcleo firme, cinturón protector, heurística positiva y negativa, contrastaciones positivas y negativas, programas progresivos y programas regresivos. Las contrastaciones positivas son aquellas en las que las predicciones hechas a partir del cinturón protector de hipótesis se cumplen y, por tanto, se constituyen en apoyo empírico del correspondiente programa, haciéndolo progresivo. En caso contrario, el programa comienza a hacerse regresivo hasta que es abandonado; abandono debido a que sus formuladores y sostenedores fallecen. Lakatos es también reconocido como uno de los epistemólogos historicistas.

Para completar la fundamentación epistemológica de nuestra propuesta, es preciso referenciar el punto de vista francés, sobre todo el de Gaston Bachelard, cuya obra se desarrolla entre 1938 y 1953. Este epistemólogo e historiador ubica sus análisis críticos, en las elaboraciones de la física y la química de principios del siglo xx. De ellas afirma que rompen con el conocimiento vulgar o común y cotidiano, puesto que generan una nueva racionalidad acerca de la naturaleza, que se aparta de todas las concepciones empiristas que venían dominando, incluyendo las aproximaciones positivistas (Bachelard, 1979). Una categoría epistemológica a la vez que pedagógica de Bachelard, es la de obstáculo, al precisar que son los conocimientos mal elaborados los que se levantan como prejuicios que impiden romper con el pasado, para adentrarse en la nueva racionalidad establecida por estas ciencias de las primeras décadas del siglo xx (Bachelard, 1972).

Bachelard en sus estudios acerca del desarrollo de las ciencias, sostiene que para explicar este desarrollo se debe acudir a la categoría de ruptura epistemológica. Cada ruptura en la época en que se sucede, produce una especie de mutación del pensamiento y de la actividad científica. Esto en razón que se abandonan las ideas y procesos anteriores, para concentrarse en los problemas que se deriven 
de la formulación de la nueva racionalidad. Esta idea la extrae de sus estudios en torno a los cambios históricos en las definiciones de los conceptos científicos o de las conceptualizaciones que al respecto, los han caracterizado entre las diversas rupturas (Bachelard, 1984). Acude igualmente a la categoría de perfil epistemológico, para estipular que cada concepto en particular, a través de sus diferentes definiciones, han pasado por concepciones propias del realismo ingenuo, el empirismo claro y positivista, el racionalismo completo, hasta ser comprendidos en el interior de un racionalismo discursivo, en el que el pensamiento y la actividad científica se libera de toda representación empirista. En este sentido, Bachelard no elabora una filosofía general de las ciencias.

En sus análisis, establece la necesaria relación entre el desarrollo de las ciencias y el de las tecnologías, demostrando la estrecha relación histórica entre ellas. De conformidad con ese perfil epistemológico, Bachelard afirma que todo concepto se hace mucho más científico, en la medida en que sea más fuertemente tecnológico. Una tecnologización que es comprensible en la perspectiva de que los coeficientes de las ecuaciones diferenciales que atrapan en su racionalidad discursiva los fenómenos, solo son materializables si se piensan en términos de las propiedades fisicoquímicas de los materiales, que hacen factible la construcción de los instrumentos o tecnofactos requeridos (Bachelard, 1979). Una lectura acerca de lo que este epistemólogo propone, conduce a pensar que, debido a esta estrecha relación entre la física y la química de las primeras décadas del siglo xx y las tecnologías, permite afirmar que estas últimas se hallan sometidas, en sus autonomías relativas como saber, también a rupturas epistemológicas.

Esa relación entre la física, la química y las tecnologías la explicita en el hecho de que la actividad científica ya no está sometida a la mirada propia del empirismo ingenuo, para el que lo observado, es lo real. Esa relación es la que hace que dicha actividad sea constructora de realidades. En otras palabras, estas ciencias de la naturaleza trabajan sobre lo real construido a partir de la racionalidad discursiva a la que los científicos han llegado (Ba- chelard, 1984), Entonces los resultados obtenidos en esa trama tecnológica que es todo experimento en ciencias; es decir, una realidad fenomenotécnica (Bachelard, 1979). Cualquier detención del pensamiento científico en la cosa observada, es un quedarse en concepciones ya superadas, tanto empiristas como positivistas.

\section{Hacia una epistemología acorde con el nuevo milenio}

A finales del siglo pasado, las sociedades han comenzado por plantearse una epistemología que vaya más allá de hacernos comprender como se han establecido a través de toda la historia de la humanidad las ciencias, sus métodos, como sus actores y sus públicos, creo que a estas alturas ya es claro el conocepto de comunidad académica y comunidades de especialistas. A este largo proceso que nos llevo a cambiar el paradigna, ya no solo de la construcción y elaboración de los conocimientos, sino que dados los escenarios en los que nos estamos desarrollando donde la preocupacion central ya no radica en la elaboración del conocimiento solamente, sino en la puesta en escena de los productos derivados de estas construcciones. A este respecto, debemos ir más allá, debemos pensar que la epistemología contemporánea una vez establecida la necesidad de incorporar una responsabilidad social a los desarrollos cientificos y tecnológicos que poco a poco se han ido apropiando de nuestra canasta familiar, debemos plantearnos una epistemología en aras de cómo elaboran los conocimientos nuestros ciudadanos. Debemos a este respecto precisar, que la concepción de ciencia y tecnología que predomina en este momento no es la misma que Kuhn, Lakatos, Popper, entre otros, hacian referencia en sus escritos, sin desmeritar sus trabajos si queremos hacer hincapie, en la necesidad de cambiar o perfilar nuestros supuestos epistémicos, sobre todo si lo que promulgamos es un cambio cultural en la formación de ciudadanos y de nuevas generaciones que ya no se enfrentan a la necesidad de hacer comprender que la ciencia se construye en equipo, creemos, o mejor queremos creer que el mito de los genios que nos vendieron en el siglo pasado es cosa del pasado. 
Si partimos de la explosión incontrolable de los productos de la ciencia y la tecnología que nos abruman y nos envuelven bajo premisas económicas, de una supuesta calidad de vida que nos ha llevado al uso y abuso de los recursos naturales, que nos ha trasformado nuestra forma de actuar, pensar y de relacionarnos los unos con los otros, esto nos lleva a pensar en que todos estos inventos que hoy son parte de nuestro que hacer diario, nunca midieron las consecuencias, pasamos de la revolución cientifica que solucionaba problemas producto de las necesidades sociales, a una ciencia y tecnología que intenta mitigar los debastadores efectos causados sobre la, a una ciencia y tecnología que busca desesperadamente la prevensión. En este sentido, es que queremos promover una nueva epistemología, que no solamente este basada en la historia de la ciencia, ya que hemos ido dejando relegada la historia de las tecnologías. Si acudimos a la historia de las ciencias, es la física la primera que se elevó a la categoría epistemológica de ciencia (Assis, 1998). Kuhn, T. S. (1972), establecia, que todo análisis epistemológico ha de basarse en una revisión histórica. Lakatos, I. (1983), estipula que toda mirada sobre la historia de las ciencias, está epistemológicamente posicionada. Por tanto habría tantas versiones sobre un mismo hecho científico, cuantas revisiones se realicen. En consecuencia, no habría una historia de la ciencia, sino distintas reconstrucciones: las empiropositivistas, las racionalistas, las deductivistas y demás; en otra palabras, tampoco resultaría sostenible hablar de fundamentos epistemológicos sino tenemos en cuenta que hablar de una historia verdadera de las ciencias hay que agregarle no solo la influencia social, sino que debemos plantearnos unos fundamentos epistémicos que incluyan al menos una versión histórica de las tecnologias. Todo esto con el fin de proponer una nueva versión epistemológica que busque y fundamente esta intrinseca relación entre las ciencias y las tecnologías, ya que si vemos los términos han ido incorporándose poco a poco indistintamente; a este respecto Fourez, en su libro Afabetización científico tecnología, las llama un matrimonio de conveniencia, ya que se unen y se separan dependiendo el contexto y la intencionalidad que lleven. Nosotros creemos que la diferencia histórica, social y cultural de las ciencias y las tecnologías podría remontarse a la historia del computador ya que es allí al interior de los grupos de investigación de universidades como Harvard, en la que participaban matemáticos, físicos, ingenieros, técnicos entre otros, que nacen los primeros prototipos considerados en su dia como avances científicos $\mathrm{y}$ es en este momento cuando los gobiernos, los responsables de obteber beneficios económicos a las investigaciones, ya que empiezan a comercializarse y a venderse y es allí donde creemos que se divorciaron las ciencias y las tecnologías, porque el conocimento científico en este momento se separa del tecnológico para continuar siendo universal y deja la tecnología de ser una mera aplicación para convertirse en un conocimiento patentable, como una mina que hay que explotar y ponerle un valor. La tecnología se volvió un negocio, puede que usted amable lector al leer estas lineas piense que las patentes ya llevaban un par de décadas, pero creemos que la ruptura social entre la ciencia y la tecnología podríamos remontarla a este episodio histórico, ya no solo primaba el afán del hombre por conquistar la naturaleza sino por dominarnos los unos a los otros. Las sociedades y las clases sociales ahora no solo se medían por sus conocimientos en las artes, las ciencias y las letras, sino comenzar a dividirnos por productos tecnológicos. Si pensamos en la sociedades actuales comparadas con las de los siglos pasados en las que se diferenciaban ,no solo por sus grandes extensiones de tierra, sino por poseer una cultura - recordemos que una persona culta era una persona cultivada- que podría hablar y profundizar en varios temas. Ahora las clases más prestantes no son las más cultas, sino las más adineradas, medidas por su gran poder adquisitivo calculado en artilujios tecnológicos, un carro moderno, un celular de última tecnología, una casa dotada de toda clase de dispositivos tecnológicos, llamadas por algunos casas inteligentes, entre otros. Esta gran diferencia de nuestras sociedades, es la que nos reafirma más en la necesidad de una alfabetización científico tecnológica y cultural, que vaya más allá de la simple información de los sistemas complejos científicos y tecnológicos que nos rodean, basada en una nueva epistemología que tenga en cuenta las diferentes versiones de estas complejas relaciones entre ciencia y tecnología, que se fundamente en la filosofía y la sociología no solo 
de la ciencia sino también de la tecnología. Esto nos llevaría a asumir posturas realmente distintas a las planteadas desde las propuestas histórico-epistemológicas de Popper, Kuhn y Lakatos. Ya en el siglo xxi, se admite que la estructura conceptual y metodológica de la ciencia no ha de seguir siendo el criterio para juzgar el estatuto de las tecnologías cuya construcción histórica y los resultados de sus investigaciones son distintos; además que los objetos de saber, los lenguajes y las intencionalidades de producción de sus comunidades de especialistas, son diferentes. Pero a su vez relacionados, la verdad es que nos queda un largo camino por recorrer y como ya lo mencione al principio no es la intención de este trabajo desarrollar una propuesta epistemológica para las relaciones científicas y tecnológicas, tan solo queremos dejar claro que para poder plantear una nueva alfabetización científico tecnológica y cultural es necesario romper con los lazos de las fundamentaciones epistemológicas del siglo pasado e ir más allá, teniendo encuenta los nuevos problemas, sociales, culturales y educativos que nos han dejado las investigaciones científicas y tecnológicas y sus productos.

\section{De la alfabetización científica y tecnológica a la alfabetización científica tecnológica y cultural}

La alfabetización inicial instaurada en el siglo XIX, entre sus objetivos no solo era la culturización de la sociedades, a partir del dominio básico de la lectura y la escritura, sino también, pretendían que la clase obrera supiera leer y escribir para que pudieran proporcionar una mano de obra más apta y cualificada. La clase obrera, porque consideraba que las instrucción, seria la llave de la emancipación. Así de esta manera fue que la escuela poco a poco llegó a ser obligatoria (Fourez, 1994). Durante largo tiempo los investigadores se han preguntado sobre los efectos de aquella alfabetización, que comenzó hace más de un siglo como una necesidad y se convirtió en obligatoriedad. De igual forma ha ido sucediendo poco a poco con los ciudadanos de este siglo, poco a poco aprender ciencia y tecnología está pasando de ser una necesidad a una obligación, necesitamos ciudadanos cualificados que les den la oportunidad no solo de adquirir algunos concep- tos básicos, sino que además, lleguen a ser buenos consumidores, lectores de publicidad, críticos y consientes de los riesgos a los que nos enfrentamos cada día por haber abusado de la naturaleza y de sus recursos. Necesitamos realmente brindarles la oportunidad de una apertura a la sociedad, de darles la libertad de elegir sobre el futuro de su pueblo y hasta que punto seguir produciendo y generando nuevos inventos científicos y tecnológicos.

\section{La alfabetización científica tecnológica y cultural}

B. Marco-Stiefel (2001) sostiene que los objetivos de una alfabetización científica fueron formulados en 1958 por Paul DeHart Hurd, aun cuando está preocupación se hallaba ya presente a finales del siglo XIX, cuando autoridades y científicos inician la presión para que las ciencias se conviertan en contenidos curriculares para la educación básica y secundaria. Se suelen traer a cuenta las palabras del físico francés, Paul Langevin, quien en una conferencia de 1926, sobre "el valor educativo de la historia de las ciencias" sostenía que "en gracia del papel jugado por la ciencia en la liberación de los espíritus y en la afirmación de los Derechos del Hombre, el movimiento revolucionario hace un esfuerzo significativo por introducir la enseñanza de las ciencias en la cultura general y conformar esas humanidades modernas que aún no hemos logrado establecer" (Calatayud, Carbonell, Carrascosa, A., Furió, M., Gil, P., Grima, J., et al. 1986, Gallego, T. 2002).

Al respecto se señala que entre la comunidad de especialistas en didáctica de las ciencias, no existe un acuerdo en torno a lo que se quiere significar con alfabetización científica, aun cuando parece inscribirse en la habitual idea que alfabetizar es enseñar a leer y a escribir (Marco-Stiefel, 2001). Una aproximación podría ser la de enseñar a leer, a escribir y hablar en el lenguaje conceptual y metodológico de cada uno de los modelos de las ciencias de la naturaleza que, como saber escolar, se hacen objeto de trabajo didáctico y pedagógico en el aula. Pero esta afirmación caería en el entramado de una educación científica que, para la autora de este libro es distinta de una educación en ciencias para todo el 
alumnado que ha de ejercer en el entramado social, político y cultural de su entorno, su ciudadanía.

Interpretando los lineamientos de Fouréz, G. (1994), ese enseñar a leer, a escribir y hablar desde el lenguaje conceptual y metodológico de los modelos científicos, curricularmente tendría que apartarse de aquellas erradas intencionalidades, que persiguen que los estudiantes se hagan científicos o aspiren a serlo, desde sus primeros contactos con la historia y la epistemología del carácter de la actividad científica; una falacia que le quitaría los objetivos a la educación universitaria, así esta sea napoleónica.

Se piensa que la alfabetización científica tecnológica y cultural, como una formación de los ciudadanos acorde con en el mundo que viven hoy en día y que les tocará vivir, afectado por los productos de las investigaciones científico-tecnológicas, requiere de un esfuerzo intelectual diferente al habitual, cuyos fundamentos han estado marcados por las aproximaciones epistemológicas empiropositivistas y las concepciones de una ciencia como producto, olvidando y alejandose de las fundamentaciones y reconstruciones de la tecnología. Se requiere que todos hagamos una revisión de sus nuestras convicciones acerca de la historia de la construcción del conocimiento científico y de sus estrechas relaciones con el conocimiento tecnológico, sobre todo en la perspectiva del actual problema del calentamiento global y del inminente agotamiento de los recursos naturales.

En este orden de ideas, la necesidad de una lectura critica y sistematica acerca de cómo afectan las relaciones sociales la adquisición en el mercado de los productos de las investigaciones científico-tecnológicas. Se trata de alfabetizar a los ciudadanos para que se enfrenten racionalmente a la publicidad que los introduce en una conducta consumista, hasta cierto punto irresponsable, en la que todo objeto comprado, más temprano que tarde se convertirá en basura y elemento contaminante del entorno. En alguna película vista en televisión por la responsable de este texto, le llamó la atención que el protagonista masculino frente a la oferta que la protagonista femenina le hacía de consumir unas moras cuyos frutos se daban en su jardín, le manifestó: "he optado por no consumir ningún producto que en la presentación de su empaque no tenga la fecha de vencimiento". Es, por tanto, una muestra de lo que podría ser el comportamiento de un ciudadano científica y tecnológicamente alfabetizado.

El que se hace urgente hoy es el que expresa el convencimiento de no usar más el carro o coche individual, para no contribuir al aumento de la cantidad de dióxido de carbono en la atmósfera planetaria, de tal manera que se quiere heredar a los nietos un ambiente en el que puedan disfrutar de la belleza de los paisajes que le dieron sentido a la existencia terrenal de los abuelos. Por ahora, predicar el empleo del transporte masivo.

El otro problema asociado a la necesidad de una alfabetización científica tecnológica y cultural tiene que ver, consecuentemente, con las decisiones que el ciudadano común ha de tomar cuando va a los supermercados o en las tiendas online en los que del mismo producto que requiere hay diferentes ofertas producidas industrialmente. La conducta más elemental es la de adquirir aquellos cuyo precios son accesibles para su economía particular; actitud esta en la que no se halla presente un análisis de los contenidos bioquímicos y procesamientos del producto, como tampoco su obsolescencia. En este comportamiento como consumidor se tiene una población que es analfabeta funcional que se deja incluir inconcientemente en un mundo afectado por la publicidad de los productos de las investigaciones científico-tecnológicas.

Hoy es innegable que esa alfabetización como proceso inicial en la formación de las nuevas generaciones, ha de dirigirse hacia la concientización del problema del cambio climático y del calentamiento global, ya que son estas nuevas generaciones, las que vivirán la "catástrofe" que ocurrirá, de seguir con los comportamientos actuales, cuando la concentración de $\mathrm{CO}_{2}$ alcance el denominado punto de no retorno. En virtud de que esta temática es multidisciplinaria, se requiere de un esfuerzo didáctico para hacerla contenido curricular, para convertirla en ciencia escolar, específicamente en 
los niveles iniciales de la educación. Para reiterarlo, Flannery, T. (2008) hace un llamado en el que se hace urgente que todos los terrestres desarrollen una cruzada contra el calentamiento global.

Obsolescencia Incluso podría afirmarse la necesidad de un cambio en lo que respecta a la formación inicial de los profesores de ciencias, quienes son formados en concepciones histórico-epistemológicas de un pasado ya lejano. Como lo afirmara T. S. Kuhn (1972), enseñan historia de las ciencias sin seguir las metodologías de los historiadores. En el nivel universitario, al entrenamiento en el empleo de metodologías estandarizadas. Se subraya al respecto, que el colectivo de especialistas interesados en socializar el problema del calentamiento global, se han ocupado de escribir libros acerca de la historia de cómo dicho problema se convirtió poco a poco en un paradigma. Estas reconstrucciones históricas constituyen un buen punto de partida para transponer dicho problema en ciencia escolar.

\section{Bibliografia}

Assis, A. K. T. (1998). Newton e suas grandes obras: O Principia e o Óptica. En: Linguagens, leituras e ensino da ciencia, De Almeida, M. j. P. M. y Da Silva, H. C. (Orgs.). Campinas: Mercado de Letras.

Deboer, G. B. (2000): Scientific Literacy: Another Look at its Historical and Contemporary Meanings and its Relationship to Science Education Reform. En: Journal of Research in Science Teaching. 37 (6): 582-601.

Barona, J. L. (1994). Ciencia e historia. Debates y tendencias en la hstoriografía de la ciencia. Valencia: Guada

Bachelard, G. (1972). La fomación del espiritu científico. Buenos Aires: Siglo XXI.

Bachelard, G. (1976). El materialismo racional. Buenos Aires: Paidos.

Bachelard, G. (1979). El racionalismo aplicado. Buenos Aires: Paidos.
Bachelard, G. (1984). La filosofía del no. Buenos Aires: Amorrortu.

Bacon, F. (1980). Novum organum. México: Porrúa.

Barona, J. L. (1994). Ciencia e historia. Debates y tendencias en la hstoriografía de la ciencia. Valencia: Guada.

Braslavsky, B. (2004). Enseñar a entender lo que se lee. La alfabetización en la escuela y en la familia. México: Editorial Fondo de Cultura Económica.

Bybee, R. (1997). Towards an Understanding of Scientific Literacy. En: GRAEBER, W., BOLTE, C. (Eds.) Scientific Literacy. Kiel: IPN.

Bybee R. W. (1991): Planet Earth in Crisis: How Should Science Educators Respond?, En: The American Biology Teacher. 53 (3): 146-153.

Caldin, E. F. (2002). The Structure of Chemistry In Relation of the Philosophy of Science. International Journal for Philosophy of Chemistry, 8 (2): 103-121. Disponible en: http://hyle.org/ journal/issues/8-2/caldin.htlm

Comte, A. (1984). Curso de filosofía positivista (lecciones 1 y 2). Discurso sobre el espíritu positivista. Barcelona: Orbis.

Eslava, J. C. (2004). Tensiones y confluencias: Una mirada fugaz al triple legado de los estudios histórico-sociales sobre la ciencia. En: Revista Colombiana de Sociología. 23: 159-180.

Fensham, P. J. (2002): Time to Change Drivers for Scientific Literacy. En: Canadian Journal of Science, Mathematics and Technology Education. 2 (1): 9-24.

Fouréz, G. (1994). Alfabetización científica y tecnológica. Buenos Aires: Calihue.

Gallego T., (2002) La imagen de la ciencia a traves del comic. Tesis Doctoral, Universitat de Valencia. 
Gieré, R. N. (1990). Explaining Science .Chicago: University of Chicago Press.

Hanson, N. R. (1977). Patrones de descubrimiento, observación y explicación. Madrid: Alianza.

Kuhn, T. S. (1972). La estructura de las revoluciones científicas. México: Fondo de Cultura Económica.

Langevin, P. (1926): La valeur éducative de l'histoire des sciences. En: Bulletin de la Société Française de Pédagogie No 22.

Lakatos, I. (1983). La metodología de los programas de investigación científica. Madrid: Alianza.

Lombardi, O. (1998). La noción de modelo en ciencias. Educación en Ciencias. 2, (4): 5-13.

Marco, B. (2000). La alfabetización científica. En: Perales, F. Y., Cañal, P. (Eds.): Didáctica de las Ciencias Experimentales. Alcoi: Mar- fil.

Mosterín, J. (1978). La estructura de los conceptos científicos. Investigación y Ciencia. 16: 82-93.

Popper, K. (1962). La lógica de la investigación científica. Madrid: Tecnos.

Restivo, S. (1992). La ciencia moderna como problema social. En: Fin de Siglo. 3: 20-39.
Scerri, E. R. (1997). Has the periodic table successfully axiomatizad. Erkenntnis. 47: 229-343.

Scerri, E. R. (2001). The new philosophy of chemistry and relevance to chemical education. Chemistry Education: Research and Practice in Europe. 2. (2): 165-170.

Scerri, E. R. (2003). Philosophical confusion in chemical education research. Journal of Chemical Education. 80. (5): 468-474.

Tomasi, J. (1999). Towards "chemical congruence" of the models in theoretical Chemistry. International Journal for Philosophy of Chemistry. 5. (2): 79-115. Disponible en: http://hyle.org/ journal/issues/5-2/tomasi.htlm

Vilches A., Solbes, J. y Gil, D. (2004). ¿Alfabetización científica para todos contra ciencia para futuros científicos ? En: Revista Alambique. 41: 89-98.

Vessuri, H. M. C. (1992). Perspectivas recientes en el estudio social de las ciencias. En: Fin de Siglo. 3: 40-52.

Zahar, E, (1982). Experimentos cruciales. Estudio de un ejemplo. En: Progreso y racionalidad en la ciencia, Radnsky, G., Anderson, G., Feyerabend, P., et al. (Eds.)70-94. Madrid: Alianza. 